\title{
Daidzein plus isolase associated with zinc improves clinical symptoms and quality of life in patients with LUTS due to benign prostatic hyperplasia: Results from a phase I-II study
}

\author{
Daniele Tiscione $^{1}$, Luca Gallelli $^{2}$, Irene Tamanini ${ }^{1}$, Lorenzo Giuseppe Luciani ${ }^{1}$, Paolo Verze ${ }^{3}$, \\ Alessandro Palmieri $^{3}$, Vincenzo Mirone ${ }^{3}$, Riccardo Bartoletti $^{4}$, Gianni Malossini ${ }^{1}$, Tommaso Cai ${ }^{1}$ \\ ${ }^{1}$ Department of Urology, Santa Chiara Regional Hospital, Trento, Italy; \\ 2 Department of Health Science, School of Medicine, University of Catanzaro, Catanzaro, Italy; \\ ${ }^{3}$ Department of Urology, University of Naples, Federico II, Naples, Italy; \\ ${ }^{4}$ Department of Urology, University of Pisa, Pisa, Italy.
}

\begin{abstract}
Summary Objective: In the last years there is a growing interest in nutraceutical substances that seems able to improve clinical symptoms in patients with lower urinary tract symptoms (LUTS) due to benign prostatic hyperplasia (BPH). In this paper, we evaluated both efficacy and safety of a combination of daidzein with isolase and zinc in patients with LUTS due to BPH.

Materials and methods: In a phase I-II study clinical trial we enrolled patients with clinical and instrumental diagnosis of LUTS associated to BPH that received a six-month treatment with a combination of daidzein with isolase and zinc (1 tablet/day). Clinical, laboratory and instrumental analyses were carried out at the time of admission (TO) and 6 months after the ending of the treatment (T1). The Italian version of International Prostatic Symptom Score (IPSS), International Index of Erectile Function (IIEF-5) and Quality of Well-Being (QoL) questionnaires were used. The development of adverse drug reactions (ADRs) and drug interactions (DDIs) were recorded using the Naranjo scale and drug interaction probability scale. Student's t test and Anova test were used for statistical analysis, and the threshold of statistical significance was set at $P<0.05$.

Results: We enrolled 71 patients, 62 (87.3\%) completed the follow-up and we documented a significant differences between T0 and T1 in terms of IPSS [21.5 \pm 1.2 vs $16.2 \pm 1.5$; $(-4.8) ; p<0.001], C_{\max }[9.7 \pm 3.7 v s 15.3 \pm 2.5 ;(+5.6) ;$ $p<0.001]$ and $Q o L[0.56 \pm 0.15$ vs $0.84 \pm 0.19 ;(+0.28)$; $p<0.001]$. In contrast, no significant difference were recorded in terms of IIEF-5 [ $p=0.50]$ and PSA [ $p=0.67]$. Finally, we did not record any significant ADRs or DDIs during the study. Conclusions: In this study, we documented that a combination of daidzein with isolase and zinc, reduces the clinical symptoms of LUTS and improves the quality of life in patients with $B P H$, without the development of ADRs or DDIs.
\end{abstract}

KEY WORDS: BPH; Zinc; LUTS; Equol; Isoflavones; Medical treatment.

Submitted 20 January 2017; Accepted 18 February 2017

\section{INTRODUCTION}

Benign prostatic hyperplasia (BPH) is one of the most common diseases affecting the elderly male and is the most common clinical condition among all urological outpatients (1). BPH is characterized by clinically rele- vant lower urinary tract symptoms (LUTS) that can reduce the quality of life (QoL) leading to serious clinical manifestations, e.g. acute urinary retention, urinary incontinence, recurrent urinary tract infection, obstructive uropathy, up to surgery (2). To date, two main categories of drugs are prescribed to treat patients with symptomatic BPH: 1) $\alpha 1$-adrenoreceptor antagonists that improve the dynamic component of urination (activation of bladder smooth muscles) and relieve the symptoms (3); 2) 5-ARIs that block the conversion of testosterone to dihydrotestosterone, and deprive the prostatic tissue of trophic androgenic influence (3). The guidelines for BPH/LUTS recommend a combination therapy with $\alpha$-blockers and 5ARI in patients with moderate-tosevere LUTS and enlarged prostates (3). Unfortunately, these treatments may be related with the development of ADRs that can induce the discontinuation of the current therapy with an impaired of both clinical symptoms and quality of life (4). Even if the current therapeutic strategies for the management of patients affected by LUTS associated to BPH are able to both control the progression of the disease and relief the symptoms, their use is related with the development of adverse drug reactions (ADRs) particularly on sexual function (5). In a recent meta-analysis Liu and co-workers, evaluating the effects of $5 \alpha$-Reductase inhibitors (5ARIs) on sexual function in men with $\mathrm{BPH}$, documented that both dutasteride and finasteride compared with placebo were associated with the development of sexual dysfunction (6). The development of ADRs represents a common problem during drug administration and represents the most common cause of low adherence to the treatment (7). In the last years, there is a grow interest for nutraceutical agents in several clinical manifestations for several reasons, e.g. low side-effect and costs, high level of adherence and a low rate of efficacy of standard treatments with subsequent patient disappointment and drop-out (8-9). Several nutraceutical products for the management of BPH have been commercialised in Italy (10) and between these, a new nutraceutical is $M I G A R^{\circledR} M G$, an association between daidzein, isolase and zinc. The effects of each component have been well-reported in literature. 
Daidzein is a soy isoflavone, after oral intake it crosses the enterocytes, is reduced to S-(-)equol through the intermediate dihydrodaidzein, and then is converted by deoxygenation to yield the bioactive S-(-)equol by intestinal bacteria (11). Several bacteria are able to perform this reaction and one of these, Lactococcus garvieae, was found in some Italian cheeses and has been used to produce the first natural S-(-) equol-containing nutraceutical (12). The bioactivation of daidzein to S-(-)equol is related to gender, race and age and requires: specific equol-producing bacteria and an optimum intraluminal conditions in terms of redox potential for the above described reactions (13). Therefore, the aim of this study is to evaluate the efficacy and safety of MIGAR ${ }^{\circledR} M G$ Retard in patients affected by LUTS related to BPH.

\section{Methods}

\section{Study design}

We performed a non-sponsored phase I-II study in a single urological institution between January to June 2016. The study was conducted according in line with Good Clinical Practice guidelines and with the ethical principles of the Declaration of Helsinki. Before the beginning of the study, all participants signed the written informed consent. No placebo run-in period was performed.

\section{Experimental protocol}

Patients with clinical and instrumental diagnosis of moderated-severe LUTS related to BPH were recruited. We considered patients with moderate-severe LUTS and with an International Prostatic Symptom Score (IPSS) score $>8$. At the time of admission (T0), the patients underwent selfadministered baseline questionnaire [IPSS, International Index of Erectile Function (IIEF-5) and Quality of Well-Being (QoL)], urological examination with history interview, prostate ultrasound and uroflowmetry $\left(\mathrm{C}_{\max }\right)$ with evaluation of post-voided residual volume (PVR), in accordance with the procedure described in EAU guidelines (3). All patients who met the inclusion criteria were enrolled and received 1 tablet/day of a combination of daidzein with isolase and zinc (MIGAR ${ }^{\circledR} M G$ Retard). The follow-up was performed 6 months after the end of therapy (T1) when patients underwent IPSS, IIEF-5QoL questionnaires and urological examination with laboratory and instrumental examinations.

\section{Inclusion and exclusion criteria}

Patients male who were $>45$ years, sexually active, with a maximal urinary flow rate $\left(\mathrm{C}_{\max }\right)<15 \mathrm{~mL} / \mathrm{s}$ and with a post-residual voided volume $<100 \mathrm{cc}$, with IPSS $=8$ or greater, with Prostate Specific Antigen (PSA) $<4 \mathrm{ng} / \mathrm{ml}$ (or higher in presence of negative prostate biopsy) and IIEF-5 score $>21$ were enclosed in this study. On the other hand, patients with diabetes, liver, and/or renal failure, cancer, prostatitis, active urinary tract infection, chronic retention or polycystic kidney disease were excluded from the study. Moreover, we also excluded patients with urethral stenosis or anatomical abnormality interfering with the evaluation of voiding function. Patients with a history of transurethral resections of the prostate, laser therapy, or thermo therapy or with allergy to one or more compounds of MIGAR ${ }^{\circledR} M G$ Retard were also excluded. Finally, were also excluded from the study the patients that received a treatment for LUTS/BPH in the last 6 months.

\section{Questionnaires and urological examinations}

The validated Italian versions of the IPSS (14), IIEF-5 (15) and QoL (16) were self-administered to each patient at the arrival to the urological institution. The QoL scale was selected because it has been successfully applied to acute illnesses, whereas other quality of life scales, including the Short Form-36 (SF-36) Health Survey, are more suitable in chronic cases (17). Higher scores on the QoL scale reflect a higher quality of life (16). The IIEF-5 questionnaire was used for exclude all patients with concomitant erectile dysfunction and for evaluating the impact of treatment on sexual function at the end of the follow-up therapy.

Composition and characterization of the extracts used MIGAR ${ }^{\circledR}$ MG Retard

Each tablet contains Daidzein $20 \mathrm{mg}$, Genistein $20 \mathrm{mg}$, Isolase ${ }^{\circledR} 40 \mathrm{mg}$, Zinc $10 \mathrm{mg}$.

Instrumental, laboratory and urological evaluation At the time of the enrolment (TO) and during the followup (T1), all patients underwent urological examinations with the prostate volume ultrasound evaluation. Ultrasound prostate evaluation was performed during the urological examination with a trans-abdominal approach. The trans-abdominal scan was performed with the patient's supine after applying ultrasonic gel to the suprapubic regions (3). Moreover, an uroflowmetry $\left(C_{\max }\right)$ with ultrasound evaluation of post-voided residual volume was performed by using URODYN ${ }^{\circledR} 1000$ System (Medtronic Functional Diagnostics A/S.) (3). All patients were asked to do the PSA dosage. In order to exclude urinary tract infection, both leukocyte esterase and nitrite levels were measured in urine samples through dipstick assay (Bayer Multistik Pro Reagent Strips) (3).

\section{Main outcome measure}

The main outcome measure was the improvement of quality of life at the end of the whole study period, in terms of changes in IPSS, IPSS-QOL, $\mathrm{C}_{\max }$, PVR, and QoL from baseline to the evaluation point, that is, 6 months. As secondary endpoints we considered a change between T0 and T1 in the following parameters: PSA values, prostate volume, progression of disease, and surgical treatment. Clinical failure was defined as the persistence of symptoms after the treatment or the suspension of therapy for significant reported ADRs, in particular on sexual function (3). Finally, from the beginning of the study (TO) and up to its ending (T1), the development of ADRs or of drug-drug interactions (DDIs) were recorded in agreement with our previous paper (18) using the Naranjo scale and the Drug Interaction Probability Scale, in agreement with the common terminology criteria for adverse events (CTCAE) guidelines (19). Safety assessments included treatment-emergent adverse events (TEAEs) and serious AEs (SAEs). The study design is reported in Figure 1. 
Figure 1.

The figure shows the study schedule. IPSS: International Prostatic Symptom Score; IIEF-5*: International Index of Erectile Function; QoL: Quality of Well-Being. V1: visit 1, at the enrolment; V2: visit 2 at the second follow-up (6 months).

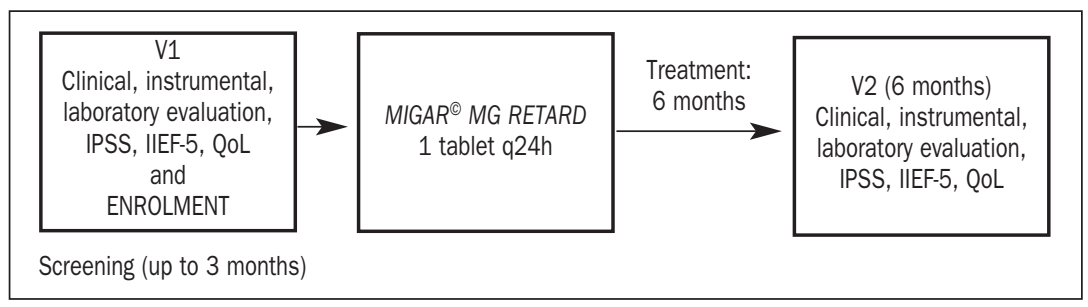

anticipated effect size, Cohen's d $=0.5$. The calculation yielded 45 individuals. At baseline, the independent sample 2tailed t-test was used to compare variables. For categorical parameters, chisquare test was applied. Changes from baseline to end of therapy were analyzed using ranked one-way analysis of variance (ANOVA) with a term for treatment group. All data are expressed as mean \pm standard deviation. The threshold of statistical significance was set at $p<0.05$. All

\section{Efficacy outcome}

The efficacy outcome was identified as a statistically significant difference between clinical data recorded at the time of admission (TO) and those recorded at the end of the study (T1).

\section{Drug adherence}

At the end of the study, the adherence to the treatment was calculated using a formula based on the number of tablets consigned at the time of admission and on the number of tablets returned unused at the end of the study.

\section{Statistical analysis}

The required sample size for the present study was calculated under the following conditions: Difference between T0 and T1, $4 \pm 1$ score points in the IPSS score; $\alpha$ error level, 0.05 two-sided; statistical power, $80 \%$; and

\section{Table 1.}

Clinical, instrumental and laboratory patient's data at the enrolment time.

\begin{tabular}{|c|c|}
\hline Patients $\left(\mathrm{n}^{0}\right)$ & 62 \\
\hline$\overline{\text { Age (mean } \pm \text { SD\#) }}$ & $65.4 \pm 5.6$ \\
\hline \multicolumn{2}{|l|}{ Marital status } \\
\hline Married & $56(90.3)$ \\
\hline Unmarried & $6(9.7)$ \\
\hline \multicolumn{2}{|l|}{ Educational qualification } \\
\hline Primary school & $31(50)$ \\
\hline High school & $20(32.2)$ \\
\hline University & $11(17.8)$ \\
\hline Sexually active (past month) & $62(100)$ \\
\hline \multicolumn{2}{|c|}{ Symptoms Score at baseline (mean \pm SD\#) } \\
\hline IPSSt & $21.5 \pm 1.2$ \\
\hline IIEF-5* & $22.1 \pm 1.8$ \\
\hline QoL† & $0.56 \pm 0.15$ \\
\hline \multicolumn{2}{|l|}{ Charlson Comorbidity Index } \\
\hline $0-1$ & $62(100)$ \\
\hline$\geq 2$ & - \\
\hline \multicolumn{2}{|l|}{ Laboratory } \\
\hline Serum totale PSA (ng/ml) & $2.47 \pm 5.6$ \\
\hline Serum creatinine (ng/ml) & $1.0 \pm 0.6$ \\
\hline \multicolumn{2}{|l|}{ Urine dipstick } \\
\hline negative & $62(100)$ \\
\hline positive & - \\
\hline \multicolumn{2}{|l|}{ Ultrasound and uroflowmetry data } \\
\hline Prostate volume (ml) & $41.5 \pm 5.8$ \\
\hline Post-voiding residual volume (ml) & $85 \pm 20.6$ \\
\hline Maximal urinary flow rate $(\mathrm{ml} / \mathrm{sec})$ & $9.7 \pm 3.7$ \\
\hline
\end{tabular}

The table shows all anamnestic, clinical and questionnaires data at enrolment SD\#: Standard Deviation; IPSS F: International Prostatic Symptom Score; IIEF-5*: International Index of Erectile Function; QoLf: Quality of Well-Being reported p-values are two-sided. All statistical analyses were performed by using SPSS 21.0 (IBM Corporation, Armonk, NY, USA), while G*Power (Institut für Experimentelle Psychologie, Heinrich Heine Universität, Dusseldorf, Germany) was used for power calculation.

\section{RESULTS}

During the study period, 71 patients were enrolled. Nine patients $(12.7 \%)$ were lost during the follow-up and excluded from the analysis, while 62 patients $(87.3 \%)$ completed the study (mean age $65.4 \pm 5.6$ years).

\section{Baseline characteristics}

History, clinical, laboratory, instrumental and questionnaires data at the time of admission are reported in Table 1.

\section{Follow-up examination}

At $\mathrm{Tl}$ we recorded a significant difference in terms of IPSS in all patients $[21.5 \pm 1.2$ vs $16.2 \pm 1.5$; $(-4.8)$; $\mathrm{p}<$ $0.001]$, and $C_{\max }[9.7 \pm 3.7$ vs $15.3 \pm 2.5 ;(+5.6) ; \mathrm{p}<$ $0.001]$, post-voiding residual volume $[85 \pm 20.6$ vs $50 \pm$ $18.5 ;(-35) ; \mathrm{p}<0.001]$, QoL [0.56 \pm 0.15 vs $0.84 \pm 0.19$; $(+0.28) ; \mathrm{p}<0.001]$. We did not record any in terms of PSA evaluation [2.47 \pm 5.6 vs $2.09 \pm 4.3 ; \mathrm{p}=0.67]$, prostate volume $[41.5 \pm 5.8$ vs $42.1 \pm 7.5 \mathrm{p}=0.61]$ and IIEF-5 [22.1 \pm 1.8 vs $22.3 \pm 1.5 \mathrm{p}=0.50]$ between T0 and T1 (Table 2).

Table 2.

Questionnaire results at the first follow-up visit (6 months).

\begin{tabular}{|lcc|}
\hline & $\begin{array}{c}\text { Baseline values } \\
\text { (SD* or \%) }\end{array}$ & $\begin{array}{c}\text { Follow-up values } \\
\text { (SD* or \%) }\end{array}$ \\
\hline Efficacy outcomes & & \\
\hline IPSS $\dagger$ & $21.5 \pm 1.2$ & $16.2 \pm 1.5$ \\
Treatment difference & $-4.8 \pm 1.1(\mathrm{p}<0.001)$ \\
\hline IIEF-5* & $22.1 \pm 1.8$ & $22.3 \pm 1.5$ \\
Treatment difference & $0.1 \pm 0.2(p=0.50)$ \\
\hline QoL§ & $0.56 \pm 0.15$ & $0.84 \pm 0.19$ \\
Treatment difference & $0.28 \pm 0.02(p<0.001)$ \\
\hline Maximal urinary flow rate $(\mathrm{ml} / \mathrm{sec})$ & $9.7 \pm 3.7$ & $15.3 \pm 2.5$ \\
Treatment difference & \multicolumn{2}{c}{$5.6 \pm 0.8(\mathrm{p}<0.001)$} \\
\hline Post-voiding residual volume $(\mathrm{ml})$ & $85 \pm 20.6$ & $50 \pm 18.5$ \\
Treatment difference & $-35 \pm 1.9(\mathrm{p}<0.001)$ \\
\hline
\end{tabular}

The table shows the mean change differences

from baseline to 6 months relative to main outcome measures.

IPSS+: International Prostatic Symptom Score; IIEF-5*: International Index of Erectile Function; QoLł: Quality of Well-Being. 


\section{ADRs}

During the study period, 1 patient (1.6\%) developed a mild ADRs (nausea) that did not require drug discontinuation or other treatments. Moreover, did not document the development of DDIs, while we recorded a 100\% adherence to the treatment and a 100\% compliance to the experimental protocol

\section{Discussion}

In this phase I-II clinical trial, we evaluated the efficacy and the safety of a new nutraceutical agent named MIGAR ${ }^{\circledR} M G$ containing daizein, isolase and zinc in the treatment of LUTS related to BPH. It has been documented that in Italy, about $50 \%$ of treatment used in patients with BPH are phytotherapies, while in Germany and other European countries, these represent the firstline treatment for mild-to-moderate LUTS in patients with $\mathrm{BPH}$. Isoflavones show a chemical structure similar to the estrogen which allows them to bind to both alpha and beta estrogen receptors, exerting estrogen-like effects and so also called as phytoestrogens (21). Whereas estrogen binds to and transactivates both estrogen receptors, isoflavones preferentially bind to and transactivate estrogen beta receptor exerciting tissue-selective effects (22) that is expressed in prostate epithelial cells and plays a role in cellular homeostasis inducing anti-proliferative, pro-differentiative (23), and pro-apoptotic effects (2324). In this concern, several clinical trials documented that isoflavones are able to suppress PSA expression in patients with prostate cancer cells. In a recent metaanalysis, Zhang et al., evaluating the effects of isoflavones on prostate cancer risk, analyzed 11.346 cases and 140.177 controls and documented that daidzein, genistein, and glycitein were associated with a reduction of prostate cancer risk with an odds ratio: 0.85, 0.87 and 0.89 , respectively (25). In agreement, in an experimental model performed in human prostate cancer cell lines $\mathrm{Lu}$ et al., recorded that s-equol has significant anti-prostate cancer activities probably related to the activation of the transcription factor Forkhead box $\mathrm{O} 3$ via an Akt-specific pathway and inhibitory effects on MDM2 expression (26). Moreover, in an experimental animal model Bae et al. reported that genistein and equol are strong inhibitors of testosterone $5 \alpha$-reductase enzyme, suggesting that isoflavones could be used in the management of patients with LUTS (27). In fact, Wong et al. documented in elderly men that the dietary intake of genistein, glycitein or daidzein was related with a significant decrease of LUTS (28). In our study, we documented that oral intake of MIGAR ${ }^{\circledR}$ MG reduces LUTS with an improvement of uroflowmetry parameters, IPSS score and QoL. Probably these improvements could be related to a potentiating of daidzein induced by isolases that seems to be able to improve the action time of equol reducing its catabolism and increasing its clinical efficacy. On the other hand the presence of zinc in this studied formulation could plays synergic effects with daidzein and isolases. In fact, a long period clinical study (4-year) documented that chronic prostate inflammation is related to both severity and progression of BPH and LUTS and zinc has antioxidant and anti-inflammatory activity and changes in prostate zinc concentrations play a role in the improvement of $\mathrm{BPH}$ (29). Take together these published data confirm our results documenting the efficacy of this new nutraceutical formulation in patients with LUTS. However, nutraceuticals are dietary supplements, and it is not necessary to demonstrate data on efficacy or safety before marketing therefore could be possible the development of ADRs or DDIs during their use. In the present study, using the Naranjo probability scale and the Drug Interaction Probability Scale, we failed to report the development of severe ADRs or DDIs during the treatment with this compound. In particular, we recorded the development of a probable mild ADR in 1 patient only but this manifestation did not induce the discontinuation of the treatment, documenting the safety of this nutraceutical. Moreover, must be underlined that enrolled patients did not develop sexual dysfunction, and the optimal sexual activity should be considered as one principal aim in the management of patients with LUTS associated to BPH (30). Previously, we documented that a better sexual quality of life is correlated with a higher overall quality of life regardless of the urinary function in patients affected by LUTS due to BPH10. Finally, in our study we documented a complete adherence (100\%) to the treatment and a complete compliance (100\%) to the experimental protocol and it may be related to the improvement of QoL. The present study has two limitations: first, the lacks of placebo arm, because in Italy ethically patients must receive a treatment during a spontaneous study, as ours; second, we evaluated the safety and efficacy of this new compound for a short time that did not allow evaluating its effects for a long time.

\section{Conclusions}

In this study, we demonstrated that combination of daidzein plus isolase and zinc (MIGAR ${ }^{\circledR}$ MG Retard) reduces the symptoms and improves the quality of life in patients affected by LUTS due to BPH, without the development of ADRs.

\section{AckNoWledgements}

We are grateful to Professor John Denton (Department of Modern Philology, University of Florence) for manuscript language revision.

\section{Contributions}

TC, DT, IT data collecting and analyzing; TC, DT, PV manuscript writing; GM, RB, AP, LG, VM supervision.

\section{REFERENCES}

1. Egan KB. The epidemiology of benign prostatic hyperplasia associated with lower urinary tract symptoms: prevalence and incident rates. Urol Clin North Am. 2016; 43:289-97.

2. Fitzpatrick JM. The natural history of benign prostatic hyperplasia. BJU Int. 2006; 97 Suppl 2:3-6; discussion 21-2.

3. Oelke M, Bachmann A, Descazeaud A, et al. EAU guidelines on the treatment and follow-up of non-neurogenic male lower urinary 
tract symptoms including benign prostatic obstruction. Eur Urol. 2013; 64:118-40.

4. Kaplan SA. Side Effects of $\boldsymbol{\alpha}$-Blocker Use: Retrograde Ejaculation. Rev Urol. 2009; 11 (Suppl 1):S14-S18.

5. Nickel JC, Sander S, Moon TD. A meta-analysis of the vascularrelated safety profile and efficacy of alpha-adrenergic blockers for symptoms related to benign prostatic hyperplasia. Int J Clin Pract. 2008; 62:1547-59.

6. Liu L, Zhao S, Li F, et al. Effect of 5 $\alpha$-Reductase Inhibitors on Sexual Function: A Meta-Analysis and Systematic Review of Randomized Controlled Trials. J Sex Med. 2016; 13:1297-310.

7. Cindolo L, Pirozzi L, Sountoulides P, et al. Patient's adherence on pharmacological therapy for benign prostatic hyperplasia (BPH)associated lower urinary tract symptoms (LUTS) is different: is combination therapy better than monotherapy? BMC Urol. 2015; 15:96.

8. Cai T, Tiscione D, Gallelli L, et al. Serenoarepens associated with selenium and lycopene extract and bromelain and methylsulfonylmethane extract are able to improve the efficacy of levofloxacin in chronic bacterial prostatitis patients. Arch Ital Urol Androl. 2016; 88:177-182.

9. Cai T, Gallelli L, Meacci F, et al. The Efficacy of Umbelliferone, Arbutin, and N-Acetylcysteine to Prevent Microbial Colonization and Biofilm Development on Urinary Catheter Surface: Results from a Preliminary Study. J Pathog. 2016; 2016:1590952.

10. Cai T, Morgia G, Carrieri G, et al. An improvement in sexual function is related to better quality of life, regardless of urinary function improvement: results from the IDIProst ${ }^{\circledR}$ Gold Study. Arch Ital Urol Androl. 2013; 85:184-9.

11. Tanaka M, Fujimoto K, Chihara Y, et al. Isoflavone supplements stimulated the production of serum equol and decreased the serum dihydrotestosterone levels in healthy male volunteers. Prostate Cancer Prostatic Dis. 2009; 12:247-52.

12. Fortina MG, Ricci G, Foschino R, et al. Phenotypic typing, technological properties and safety aspects of Lactococcus garvieae strains from dairy environments. J Appl Microbiol. 2007; 103:445-53.

13. Kenneth D, Setchell R, Clerici C. Equol: History, Chemistry, and Formation. J Nutr. 2010; 140:1355S-1362S.

14. Badia X, Garcia-Losa M, Dal-Re R. Ten-language translation andharmonization of the International Prostate Symptom Score: developinga methodology for multinational clinical trials. Eur Urol. 1997; 31:129-40.

15. Rosen RC, Riley A, Wagner $G$, et al. The international index of erectilefunction (IIEF): a multidimensional scale for assessment of erectiledysfunction. Urology. 1997; 49:822-30.

16. Kaplan RM, Bush JW, Berry CC. Health status: types of validity and the index of wellbeing. Health Serv Res 1976; 11:478-507.

17. Apolone G, Mosconi P. The Italian SF-36 Health Survey: translation, validation and norming. J Clin Epidemiol. 1998; 51:1025-36.

18. Gallelli L, Colosimo M, Tolotta GA, et al. Prospective randomized double-blind trial of racecadotril compared with loperamide in elderly people with gastroenteritis living in nursing homes. Eur J Clin Pharmacol. 2010; 66:137-44.

19. Naranjo CA, Busto U, Sellers EM, et al. A method for estimating the probability of adverse drug reactions. Clin Pharmacol Ther. $1981 ; 30: 239-245$

20. Kuiper GG, Carlsson B, Grandien K, et al. Comparison of the ligand binding specificity and transcript tissue distribution of estrogen receptors alpha and beta. Endocrinology. 1997; 138:863-870.
21. Margeat E, Bourdoncle A, Margueron $R$, et al. Ligands Differentially Modulate the Protein Interactions of the Human Estrogen Receptors alpha and beta. J Mol Biol. 2003; 326:77-92.

22. Zhang W, Makela S, Andersson LC, et al. A role for estrogen receptor beta in the regulation of growth of the ventral prostate. Proc Natl Acad Sci. USA 2001; 98:6330-6335.

23. Imamov O, Morani A, Shim GJ, et al. Estrogen receptor-beta regulates epithelial cell differentiation in the mouse ventral prostate. Proc Natl Acad Sci. USA 2004; 101:9375-9380.

24. McPherson SJ, Hussain S, Balanathan P, et al. Estrogen receptor-beta activated apoptosis in benign hyperplasia and cancer of the prostate is androgen independent and TNFalpha mediated. Proc Natl Acad Sci. USA 2010; 107:3123-3128.

25. Zhang Q, Feng H, Qluwakemi B, et al. Phytoestrogens and risk of prostate cancer: an updated meta-analysis of epidemiologic studies. Int J Food Sci Nutr. 2016; 9:1-15.

26. Lu Z, Zhou R, Kong Y, et al. S-equol, a Secondary Metabolite of Natural Anticancer Isoflavone Daidzein, Inhibits Prostate Cancer Growth In Vitro and In Vivo, Though Activating the Akt/FOXO3a Pathway. Curr Cancer Drug Targets. 2016; 16:455-65.

27. Bae M, Woo M, Kusuma IW, et al. Inhibitory effects of isoflavonoids on rat prostate testosterone $5 \alpha$-reductase.J Acupunct Meridian Stud. 2012; 5:319-22.

28. Wong SY, Lau WW, Leung PC, et al. The association between isoflavone and lower urinary tract symptoms in elderly men. Br J Nutr. 2007; 98:1237-42.

29. Jarosz M, Olbert M, Wyszogrodzka G, et al. Antioxidant and anti-inflammatory effects of zinc. Zinc-dependent NF- $\alpha B$ signaling. Inflammopharmacology 2017; 25:11-24

30. Messina R, Mirone V. Benign Prostatic Hyperplasia - An economic assessment of fixed combination therapy based on a literature review. Arch Ital Urol Androl. 2015; 87:185-9.

\section{Correspondence}

Daniele Tiscione, MD

Irene Tamanini, MD

Lorenzo Giuseppe Luciani, MD

Gianni Malossini, MD

Tommaso Cai, MD

ktommy@libero.it

Department of Urology, Santa Chiara Regional Hospital

Largo Medaglie d'Oro 9 - Trento, Italy

Luca Gallelli, MD

Department of Health Science, School of Medicine,

University of Catanzaro, Catanzaro, Italy

Paolo Verze, MD

Alessandro Palmieri, $M D$

Vincenzo Mirone, MD

Department of Urology, University of Naples, Federico II, Naples, Italy

Riccardo Bartoletti, MD

Department of Urology, University of Pisa, Pisa, Italy 